\title{
Integration of Electrical, Structural, and Anatomical imaging for the guidance of Cardiac Resynchronization Therapy
}

\author{
Uyên Châu Nguyên ${ }^{1}$, Matthijs J.M. Cluitmans ${ }^{2}$, Casper Mihl ${ }^{3}$, Justin J.G. Luermans ${ }^{2}$, MD, PhD, Bas \\ L.J.H. Kietselaer ${ }^{2}$, Sebastiaan C.A.M. Bekkers ${ }^{2,3}$, Suzanne Gommers ${ }^{3}$, Paul G.A. Volders ${ }^{2}$, Frits W. \\ Prinzen ${ }^{1}$, Kevin Vernooy ${ }^{2}$
}
Department of Physiology ${ }^{1}$, Cardiology ${ }^{2}$, and Radiology ${ }^{3}$, Maastricht University Medical Center, Maastricht, the Netherlands

\begin{abstract}
Background: Placing the left ventricular (LV) lead at a site of late electrical activation remote from scar is desired for cardiac resynchronization therapy (CRT) response.

Objective: The purpose of this study was to integrate electrocardiographic imaging (ECGi) with computed tomography (CT) derived coronary venous anatomy and delayed enhancement cardiac magnetic resonance imaging (DE-CMR) derived scar to reconstruct a pre-procedural roadmap for LV lead guidance in CRT.

Methods: Three CRT candidates with focal scar defined by DE-CMR were prospectively included. Intrinsic body surface potentials measurements (BSPM) were carried out. Inverse reconstruction using the CT heart-torso geometry were used for ECGi. Meshes of the CT coronary veins, epicardium with ECGi activation times, and DE-CMR scar were integrated.

Results: The ECGi-CT-CMR roadmap was used for CRT implantation in $2 / 3$ patients. Placing the LV lead remote from scar was accomplished in $2 / 2$ patients. Target veins from the ECGi-CT-CMR roadmap were located in a region of 80-105 ms electrical delay.

Conclusion: ECGi-CT-CMR roadmaps can be used during CRT implantation to guide LV lead placement to a coronary vein remote from scar in a region of late electrical activation, possibly improving CRT.
\end{abstract}

\section{Introduction}

Cardiac resynchronization therapy (CRT) is an effective treatment for dyssynnchronous heart failure (HF). Despite the striking effectiveness of CRT, the issue remains that a substantial group of eligible patients don't benefit from this therapy.(1) This reduced benefit has been partly attributed to a suboptimal CRT lead in the left ventricle (LV).(1) LV lead placement in a region of late electrical activation is associated with superior clinical outcomes in CRT patients.(2) However there is large inter-individual variability in the location of latest activated region exists. Furthermore, pacing in myocardial scar diminishes the effectiveness of CRT.(3) Additionally, LV lead placement is limited to the coronary venous anatomy. Thus, placing the LV lead in a coronary vein in a region of late electrical activation remote from scar is desired to optimize CRT response.

Electrocardiographic imaging (ECGi) can reconstruct the electro-anatomic activation of the epicardium based on non-invasive body surface potential measurements (BSPM) and patient-specific heart-torso geometry.(4) Delayed enhancement cardiac magnetic resonance imaging (DE-CMR) is presently considered the clinical gold standard for evaluating the presence and extent of focal myocardial scar. Contrast enhanced computed tomography (CT) has the ability to visualize coronary venous anatomy. Integration of ECGi with coronary venous $\mathrm{CT}$ and DE-CMR takes advantage of the benefit of multiple modalities by enables 3D visualization of epicardial electrical activation in relation to coronary venous anatomy and scar tissue location. This integrated 3D image can serve as a pre-procedural roadmap for CRT implantation and guide LV lead placement. The objectives of the present therefore study was (1) to integrate ECGi epicardial activation maps with DE-CMR confirmed scar and CT coronary veins pre-procedurally, (2) to use the integrated 3D image as a roadmap to guide LV lead placement to a coronary vein remote from scar in a region of late electrical activation during CRT implantation, and (3) to validate the anatomy of the coronary veins and electrical activation pattern from the pre-procedural roadmap with invasive measurements.

\section{Methods}

\subsection{Study population}

Consecutive patients referred for CRT were prospectively enrolled from March 2017 at Maastricht University Medical Center. Enrolment will continue till 
October 2017. Patients with regular contra-indications for CT or CMR were excluded. Written informed consent was obtained from each patient and the institutional review board approved the study protocol.

\subsection{BSPM and CT}

Intrinsic BSPM were carried out with ca. 200 electrodes on the patient torso, three limb leads, and a reference electrode on the lower abdomen. CT imaging was performed using a third generation dual-source scanner (Somatom Definition Force, Siemens Healthcare, Forchheim, Germany). Contrast medium was injected in a triphasic injection protocol. A non-enhanced thorax scan was performed to determine the position of all BSPM electrodes. A test bolus injection at the level of the ascending aorta was used to assess optimal delay for imaging of the coronary venous anatomy. Delay was calculated by time to peak +12 seconds. To reconstruct the torso geometry and define the position the BSPM electrodes, all BSPM electrodes were manually segmented using Seg3D v2.4. Patient-specific meshes of the epicardium and coronary veins were constructed by segmentation of LV and RV epicardial contours and coronary veins.

Table 1 Baseline characteristics

\begin{tabular}{llll}
\hline & Pt1 & Pt2 & Pt3 \\
\hline Demographics & & & \\
Age (years) & 69 & 67 & 71 \\
Gender & Male & Male & Male \\
BMI (kg/m2) & 31 & 22 & 27 \\
NYHA (II/III) & II & II & II \\
CMR & & & \\
LVEF (\%) & 28 & - & 11 \\
EDV(ml) & 387 & - & 505 \\
LV mass (g) & 150 & - & 223 \\
Scar location & I/IL/AL & - & AL//IL/I \\
Surface ECG & & & \\
Intrinsic rhythm & AF & Sinus & Sinus \\
QRSd (ms) & 134 & 152 & 138 \\
Conduction & IVCD & LBBB & IVCD \\
\hline
\end{tabular}

$\mathrm{AL}=$ anterolateral, $\mathrm{EDV}=$ end-diastolic volume, $\mathrm{I}=$ inferior, $\mathrm{IL}=$ inferolateral, $\mathrm{L}=$ lateral, $\mathrm{LVEF}=\mathrm{LV}$ ejection fraction, $\mathrm{NYHA}=\mathrm{New}$ York Heart Association functional class.

\subsection{ECGi epicardial activation map}

Inverse reconstructions were performed on the basis of the potential-based formulation of ECGi as described previously using custom software programmed in MATLAB r2016B.(5) The epicardial segmentations were re-sampled to a mesh of $<3.000$ nodes using PreView. Epicardial unipolar electrograms were reconstructed for every node by concatenating potentials over time. A window defining the onset and end of the QRS complex and T-wave for all electrograms was manually defined per patient. Activation times were automatically computed from ECG QRS onset to the steepest spatiotemporal downslope in the QRS-complex, while recovery times were regarded as the steepest spatiotemporal upslope in the T-wave window.(5)

\subsection{DE-CMR}

CMR scans were part of daily clinical routine care. Acquisition protocols and data post-processing have been described previously.(6) CMR images were acquired with a 1.5 or 3.0 T scanner (Philips Medical Systems, Best, The Netherlands). DE-CMR images were acquired with a $2 \mathrm{D}$ inversion gradient echo sequence, 7-15 minutes after intravenous bolus injection of Gadobutrol. Endocardial and epicardial borders were manually traced from shortaxis DE-CMR images using commercially available software (CAAS MRV 3.4, Maastricht, The Netherlands). Focal scar was semi-automatically quantified using the full-width-half-maximum method.

\subsection{ECGi-CMR-CT roadmap}

All meshes (CT coronary veins, CT epicardium with ECGi reconstructed activation times, and DE-CMR myocardial scar) were imported in Paraview 5.1.0. DECMR meshes were manually rotated and translated to match the coordinate system of the CT meshes using the $\mathrm{RV}$ and LV apex and LV epicardium as reference anatomic landmarks. A pre-procedural roadmap was reconstructed with integration of the coronary veins, ECGi activation map of CT epicardium, and the translated and rotated DECMR derived myocardial scar (Figure 1).

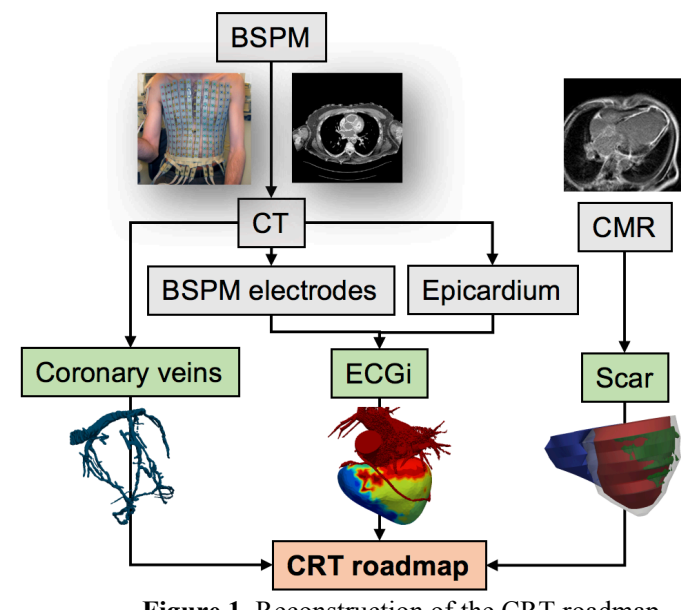

\subsection{CRT implantation}

A fluoroscopy was recorded of the CS and tributaries for visualization of coronary venous anatomy. The right 
atrial lead was placed in the right atrial appendage, while the right ventricular (RV) lead was targeted towards the RV apex. The electrophysiologist was initially asked to choose a target vein for LV lead placement based on fluoroscopy blinded for the pre-procedural roadmap. After decision making, the pre-procedural roadmap was presented to the electrophysiologist and LV lead placement was targeted towards the coronary vein remote from scar in a region of late electrical activation (preferable $>50 \%$ of QRS duration) based on the roadmap. Navigation of the LV lead to the target region was based on fluoroscopy. Quadripolar LV leads were used. The electrode with a low threshold without phrenic nerve stimulation was selected for pacing. Intrinsic electrograms at the RV and LV lead were recorded simultaneously with a precordial lead (V1) using BARD. Electrical delay from these electrograms was measured as the duration between onset of surface ECG QRS duration and the steepest downslope in the QRS window of the intra-cardiac electrogram.

Table 2 CRT implantation

\begin{tabular}{|c|c|c|c|}
\hline & Pt1 & Pt2 & Pt3 \\
\hline \multicolumn{4}{|l|}{ CT-CMR } \\
\hline Coronary veins & $\begin{array}{l}\text { AV, ALV, } \\
\text { ILV, IV }\end{array}$ & - & $\mathrm{IV}^{\mathrm{AV}, \mathrm{ILV},}$ \\
\hline Veins outside scar & AV, ALV & - & ILV \\
\hline Target veins & AV, ALV & - & ILV \\
\hline \multicolumn{4}{|l|}{ ECGi } \\
\hline $\begin{array}{l}\text { Total activation (ms, } \\
\% \text { QRSd) }\end{array}$ & $111(82 \%)$ & $\begin{array}{r}117 \\
(78 \%)\end{array}$ & $116(84 \%)$ \\
\hline $\begin{array}{l}\text { Electrical delay RV } \\
\text { apex }(\mathrm{ms}, \% \mathrm{QRSd})\end{array}$ & $45(34 \%)$ & $\begin{array}{c}36 \\
(31 \%)\end{array}$ & $48(35 \%)$ \\
\hline \multicolumn{4}{|l|}{ ECGi-CT-CMR } \\
\hline LAV & $\mathrm{AV}, \mathrm{ALV}$ & - & ILV \\
\hline Electrical delay & 105,80 & - & $103(75 \%)$ \\
\hline LAV (ms, \%QRSd) & $(78 \%, 63 \%)$ & & \\
\hline Target veins & $\mathrm{AV}, \mathrm{ALV}$ & - & ILV \\
\hline \multicolumn{4}{|l|}{ Fluoroscopy } \\
\hline Coronary veins & $\begin{array}{l}\text { AV, ALV, } \\
\text { ILV, IV }\end{array}$ & - & $\begin{array}{l}\text { AV, LV, } \\
\text { ILV, IV }\end{array}$ \\
\hline Target vein & ALV & - & LV \\
\hline Final LV lead & ALV & - & ILV, LV \\
\hline \multicolumn{4}{|l|}{ Electrical delay } \\
\hline RV lead (ms) & 58 & - & 66 \\
\hline LV lead D1 (ms) & 66 & - & 132 \\
\hline LV lead M2 (ms) & 84 & - & 148 \\
\hline LV lead M3 (ms) & 76 & - & 120 \\
\hline LV lead P4 (ms) & 94 & - & 124 \\
\hline
\end{tabular}

\section{Results}

\subsection{Study population}

Three consecutive patients referred for CRT device implantation and focal scar on DE-CMR were so far enrolled in March and April 2017 (enrolment is still open).
Patient baseline characteristics are shown in Table1.

\subsection{Roadmap reconstruction}

BSPM were carried out in all patients with an average recording time of $35 \pm 5$ minutes. CT imaging of the BSPM electrodes was performed in all patients. CT imaging of the coronary veins was performed in Patient 1 and 3, but unfortunately cancelled in Patient 2 due to a logistical error. A redo of the CT coronary veins for Patient 2 is planned in May 2017. Coronary veins shown on CT per patient are represented in Table 2. CMR was performed in Patient 1 and Patient 3. Patient 2 is planned for CMR in May 2017. CMR outcome variables are shown in Table 1, while the CT coronary veins located remote from DECMR confirmed scar are shown in Table 2. For ECGi reconstruction, BSPM from intrinsic sinus heart beats were analyzed. The CT epicardium was adjusted to a mesh of 2968, 3018, and 2805 nodes for patient 1, 2, and 3 respectively. Activation times were computed for every single node. Total activation time of depolarization of the whole epicardium per patient is shown in Table 2. Target veins based on image integration are shown in Table 2 and a representative roadmap is shown in Figure 2.

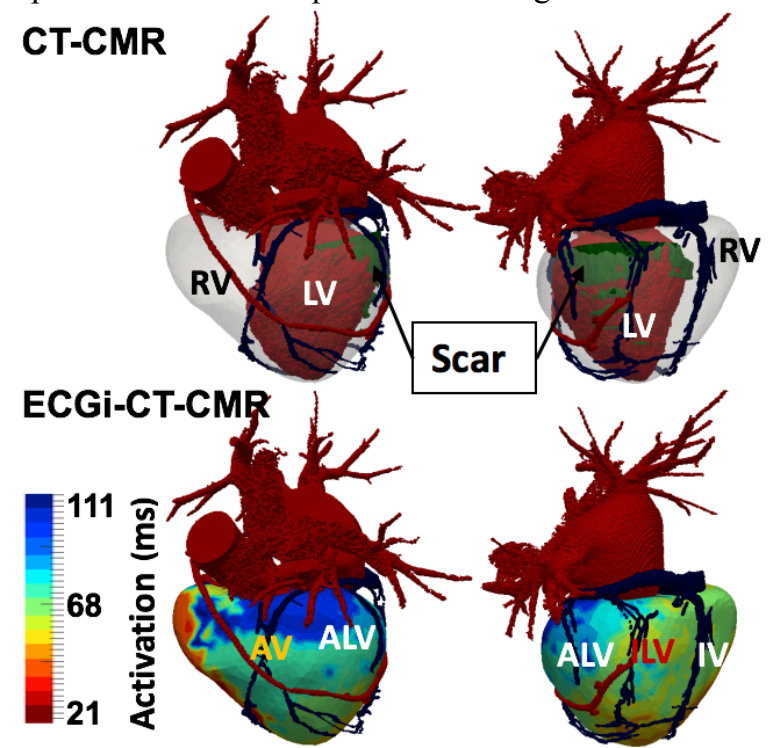

Figure 2. CRT roadmap for Patient 1. Integrated image of the CT coronary veins and DE-CMR scar (dark green) (upper row). There were four coronary veins on the CT: anterior vein (AV), anterolateral vein (ALV), inferolateral vein (ILV), and inferior vein (IV). Scar in this patient was predominantly present at the lateral wall. Only the AV and partly the ALV were located outside scar. Integrated image of epicardial ECGi, CT coronary veins, and DE-CMR scar (lower row). Note that the AV and ALV are located in an electrically late activated region.

\subsection{CRT implantation}

Patient 1 and 3 underwent a de novo CRT device implantation guided by ECGi-CT-CMR road mapping without complications. Patient 2 is planned CRT 
implantation in June 2017. Total procedural time was 120 and 180 minutes for Patient 1 and 3 respectively. Fluoroscopy time was 16 and 57 minutes with radiation doses of 237 and $914 \mathrm{mGy}$ for Patient 1 and 3 respectively.

The coronary veins on fluoroscopy were similar as found on CT for patient 1, while for patient 3 one coronary vein (lateral vein) present on fluoroscopy was not present on CT. Target veins based on ECGi-CT-CMR road mapping were similar to those based on fluoroscopy for Patient 1 and 3. Although in patient 3, the final LV lead was positioned in the inferolateral vein and an anastomotic branch of the lateral vein that was not shown on CT. Specific fluoroscopy target veins and final LV lead positions are shown in Table 2. Representative invasive fluoroscopy images are compared with the pre-procedural CT images of the coronary veins in Figure 3. ECGi and invasive recorded electrical delays are shown in Table 2.
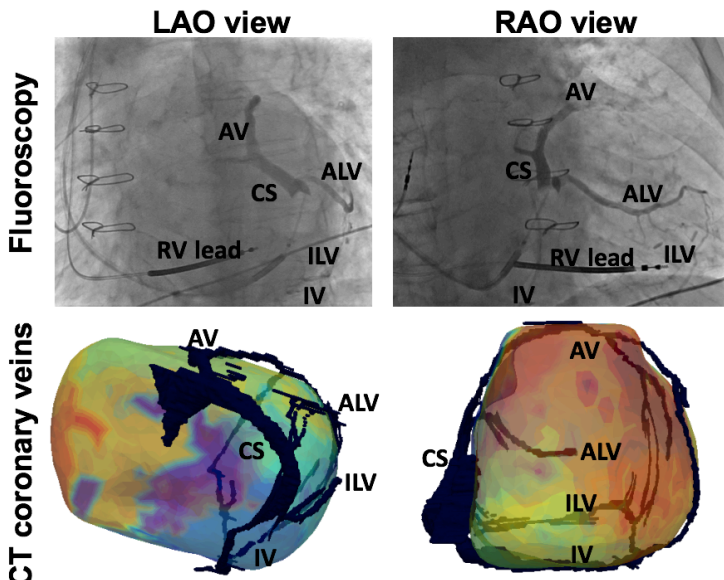

Figure 3. Fluoroscopy images of the coronary veins (upper row) in Patient 1. Pre-procedural 3D reconstruction of the CT coronary veins (lower row) integrated with ECGi in corresponding views. The preprocedural anatomy was highly similar with the anatomy derived from invasive fluoroscopy images, although the presence of collateral branches was more evident in the fluoroscopy images. Abbreviations: $\mathrm{AV}=$ anterior vein, $\mathrm{ALV}=$ anterolateral vein, $\mathrm{CS}=$ coronary sinus, $\mathrm{ILV}=$ inferolateral vein, $\mathrm{IV}=$ inferior vein, $\mathrm{RV}=$ right ventricular.

\section{Discussion}

In the present study, we demonstrated a novel approach for LV placement in CRT by integrating electrical information from ECGi with both CT derived coronary venous anatomy, and structural information on the presence and extent of myocardial scar from DE-CMR. This pre-procedural ECGi-CT-CMR roadmap was used to guide LV lead placement to a coronary vein remote from scar in a region of late electrical activation. We have shown the preliminary results of the first three patients. ECGi epicardial activation maps were reconstructed for all three patients. ECGi-CT-CMR pre-procedural roadmaps were constructed for two patients and used to guide LV placement during CRT implantation.

The electrical delay measured invasively at the RV lead was consequent lower in Patient 1 and 3 compared to the RV apex electrical delay from the ECGi activation map, which could be explained by the fact that the position of the RV lead is located at the endocardium, while the ECGi reconstructions were done at the epicardium. For patient 1 the electrical delay at the ECGi anterolateral vein (which was the final LV position) was in the ranges of the LV lead electrical delay measurements. In patient 3 the electrical delay at the ECGi inferolateral vein was lower compared to the LV lead electrical delays measured invasively. In this patient, one coronary vein (lateral vein) present on fluoroscopy was missed on CT, and the LV lead was finally placed only partially in the initial target vein explaining the slight differences in electrical delay.

Coronary venous anatomy was similar on the preprocedural roadmap with invasive fluoroscopy images in one patient. In the other patient, CT failed to identify one coronary vein in comparison to fluoroscopy. In the two patients so far, the LV lead was placed in the ECGi-CTCMR target vein. The target vein from our pre-procedural roadmap was similar to ones chosen by the electrophysiologist based on fluoroscopy, indicating that for the first two CRT implantations, clinical decision making on LV placement was not changed by the ECGiCT-CMR roadmap. Still, road mapping may still be of added value as information on the LV lead position with regard to scar and electrical substrate could provide additional insight in patient (non)response to CRT.

\section{References}

1. Leyva F, Foley PW, Chalil S, Ratib K, Smith RE, Prinzen F, et al. Cardiac resynchronization therapy guided by late gadoliniumenhancement cardiovascular magnetic resonance. Journal of cardiovascular magnetic resonance : official journal of the Society for Cardiovascular Magnetic Resonance. 2011;13:29.

2. Kandala J, Upadhyay GA, Altman RK, Parks KA, Orencole M, Mela T, et al. QRS morphology, left ventricular lead location, and clinical outcome in patients receiving cardiac resynchronization therapy. European heart journal. 2013;34(29):2252-62.

3. Wong JA, Yee R, Stirrat J, Scholl D, Krahn AD, Gula LJ, et al. Influence of pacing site characteristics on response to cardiac resynchronization therapy. Circulation Cardiovascular imaging. 2013;6(4):542-50.

4. Ramanathan C, Ghanem RN, Jia P, Ryu K, Rudy Y. Noninvasive electrocardiographic imaging for cardiac electrophysiology and arrhythmia. Nat Med. 2004;10(4):422-8.

5. Cluitmans M, Bonizzi P, Karel J, Das M, Kietselaer B, de Jong $\mathrm{M}$, et al. In Vivo Validation of Electrocardiographic Imaging. JACC: Clinical Electrophysiology. 2017; In press.

6. Nguyen UC, Mafi-Rad M, Aben JP, Smulders MW, Engels EB, van Stipdonk AM, et al. A novel approach for left ventricular lead placement in cardiac resynchronization therapy: Intraprocedural integration of coronary venous electroanatomic mapping with delayed enhancement cardiac magnetic resonance imaging. Heart rhythm. 2017;14(1):110-9.

\section{Address for correspondence.}

Ms. Uyên Châu Nguyên, Cardiovascular Research Institute Maastricht, P.O. Box 616, 6200 MD Maastricht, The Netherlands, email: u.nguyen@maastrichtuniversity.nl. 